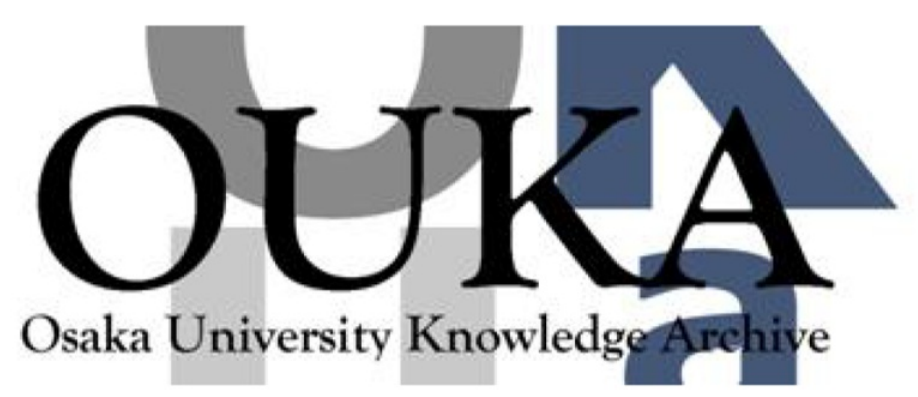

\begin{tabular}{|c|c|}
\hline Title & $\begin{array}{l}\text { Enhancement of sensitivity of } \mathrm{Pd} \text {-based } \\
\text { hydrogen-gas sensor by plasma exposure studied } \\
\text { by wireless quartz resonator }\end{array}$ \\
\hline Author (s) & $\begin{array}{l}\text { Zhou, Lianjie; Nakamura, Nobutomo; Nagakubo, } \\
\text { Akira et al. }\end{array}$ \\
\hline Citation & $\begin{array}{l}\text { Japanese Journal of Applied Physics. } 59 \\
\text { p. SKKB02 }\end{array}$ \\
\hline Issue Date & $2020-07$ \\
\hline oaire:version & AM \\
\hline URL & https://hdl. handle. net/11094/85130 \\
\hline rights & $\begin{array}{l}\text { (- } 2020 \text { The Japan Society of Applied Physics. } \\
\text { This Accepted Manuscript is avai lable for reuse } \\
\text { under a Creative Commons Attribution- } \\
\text { NonCommercial-NoDerivatives } 4.0 \text { International } \\
\text { License after the } 12 \text { month embargo period } \\
\text { provided that all the terms of the license are } \\
\text { adhered to. }\end{array}$ \\
\hline Note & \\
\hline
\end{tabular}

Osaka University Knowledge Archive : OUKA

https://ir. Library. osaka-u. ac. jp/

Osaka University 


\title{
Enhancement of sensitivity of Pd-based hydrogen-gas sensor by plasma exposure studied by wireless quartz resonator
}

\author{
Lianjie Zhou ${ }^{1}$, Nobutomo Nakamura ${ }^{2}$, Akira Nagakubo ${ }^{1}$, and Hirotsugu Ogi ${ }^{1 *}$ \\ ${ }^{1}$ Graduate School of Engineering, Osaka University, Yamadaoka 2-1, Suita, Osaka 565-0871, Japan \\ ${ }^{2}$ Graduate School of Engineering Science, Osaka University, Toyonaka, Osaka 560-8531, Japan
}

Sensitivity of hydrogen-gas sensor based on lattice expansion of palladium highly depends on the surface morphology of palladium. We find that the sensitivity can be significantly improved by exposing the as-deposited palladium film to low-power plasma. The hydrogen-gas detection was performed by a wireless-electrodeless AT-cut quartz-crystal resonator with $125-\mathrm{MHz}$ fundamental resonance frequency. It detects hydrogen gas through bending deformation of the resonator caused by the volume expansion of palladium. The surface morphology of palladium film before and after the plasma treatment were analyzed using AFM. The plasma treated palladium film exhibits rougher surface, finer grains, voids, and grain boundary extension. Such morphology and structure changes along with defects induced by the ion bombardment during the plasma treatment strongly contribute to increase in hydrogen absorption rate and then the sensor sensitivity. We further investigate the thickness dependence of the sensitivity, revealing an optimum palladium film thickness of $300 \mathrm{~nm}$.

Japanese Journal of Applied Physics 59, SKKB02 (2020)

https://doi.org/10.35848/1347-4065/ab78e2

\section{Introduction}

Hydrogen gas is a promising alternative energy carrier, and a highly sensitive, fast, and repeatable hydrogen-gas sensor is needed with growth of the hydrogen usage. Ultrasonic resonator has wide application in gas sensors. ${ }^{1,2}$ In existing hydrogen-gas sensors, palladium and its alloys have been used as the hydrogen-gas receptor materials, ${ }^{3-9}$ owing to their high selectivity and absorption ability to hydrogen gas. The sensitivity of palladium-based hydrogen-gas sensor is determined by the hydrogen atom content near palladium surface and speed of hydrogen diffusion from surface into the lattice. For most hydrogen-gas sensor, in order to achieve high sensitivity, the sensing process is performed at elevated temperatures to accelerate the absorption reaction. This approach, however, increases the difficulty of implementation and also poses a risk for practical application due to the inflammability of hydrogen gas. A method that can improve the sensitivity of hydrogen-gas sensor without largely increasing operation temperature is preferred. Another important issue is improvement of hydrogen-gas absorption behavior of palladium, because it is known that as-deposited palladium film shows poor hydrogen absorption performance, limiting the sensitivity of the palladium-based hydrogen-gas sensor. ${ }^{10,11}$

Previous studies indicate that introduction of defects in hydrogen storing materials improves their hydrogen absorption ability: A low-energy electron-beam treatment can reduce the activation energy of hydrogen bulk diffusion in titanium and enhance the hydrogen absorption ability. ${ }^{12}$ Introduction of defects by ion-beam irradiation was also used to enhance the hydrogen adsorption behavior in single-wall carbon nanotubes,${ }^{13}$ in palladium, ${ }^{14}$ and in Pd-Au alloy thin films. ${ }^{15}$ An electron-beam irradiation was also used to fabricate high sensitive $\mathrm{ZnO}$-nanofiber-based hydrogen sensor. ${ }^{16}$

We here find that a low-power plasma treatment can significantly improve the hydrogen-gas absorption ability of palladium thin films, resulting in highly sensitive hydrogen-gas sensor. Plasma has been used as an effective and econom- ical surface treatment technique. ${ }^{17}$ Its effects on materials are manifold, including chemical etching, mechanical ablation, and plasma annealing ${ }^{18,19}$ induced by electron and ion bombardment. Therefore, the surface and subsurface structure of palladium film can be modified so as to improve the hydrogen-gas absorption rate by the plasma treatment. In this research, we investigate the effect of the plasma treatment on the deposited palladium thin film using a high-frequency wireless-electrodeless quartz crystal resonator. ${ }^{20,21}$ We studied the effect of plasma treatment with different exposure time on the response and detection sensitivity of the palladium coated AT-cut-quartz hydrogen-gas sensor. We observed significant sensitivity improvement by the plasma treatment. To clarify its mechanism, we analyzed the structure change of the palladium film before and after the plasma treatment using atomic force microscopy (AFM). Furthermore, we investigated the film-thickness dependence of the hydrogen-gas detection sensitivity.

\section{Experiments}

\subsection{Fabrication of quartz resonator hydrogen sensor}

The hydrogen-gas detection sensitivity of palladium thin film is evaluated using wireless-electrodeless quartz-crystal sensor. ${ }^{22-25}$ The AT-cut quartz-crystal resonator has a rectangular parallelepiped area of $2.5 \times 1.7 \mathrm{~mm}^{2}$ and the thickness of $13.5 \mu \mathrm{m}$, exhibiting the fundamental through-thickness shearmode resonant frequency of $125 \mathrm{MHz}$. The metallic thin films were deposited using the radio frequency (RF) magnetronsputtering method: We first deposited $5 \mathrm{~nm}$ chromium and then palladium of various thicknesses from $50 \mathrm{~nm}$ to $500 \mathrm{~nm}$ on a single side of the AT-cut crystal. The thickness of the palladium film was measured by the X-ray total reflectivity measurement. ${ }^{26}$ The plasma treatment was performed in a lowvacuum plasma cleaner (PDC-001, Harrick Plasma (115V)) with the power of $30 \mathrm{~W}$ by flowing air at a pressure of 850 mTorr. 


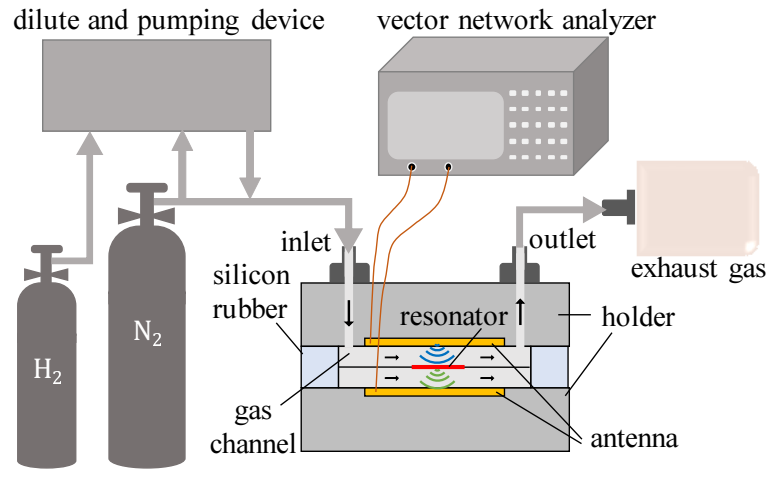

Fig. 1. Schematic of the experimental system. ${ }^{27}$

\subsection{Hydrogen-gas detection with a wireless quartz crystal resonator}

Figure 1 illustrates the homebuilt experimental system. The palladium-coated quartz resonator was lightly sandwiched by two silicon-rubber gaskets in the sensor cell. The sensor cell was placed on a heater to keep the temperature at $55^{\circ} \mathrm{C}$. (Our previous study showed that our sensor exhibited the best performance at this temperature..$^{28}$ ) We flowed high purity nitrogen gas $(99.9999 \%)$ to the sensor cell as a carrier gas at a flow rate of $100 \mathrm{~mL} / \mathrm{min}$ and the diluted hydrogen gas at a flow rate of $34.5 \mathrm{~mL} / \mathrm{min}$. They got together and entered the sensor cell with an intended hydrogen-gas concentration. The resonant frequency was monitored by the transmission coefficient (S12 parameter) by a vector network analyzer (ZNB, Rohde \& Schwarz). Because the vibration excitation and detection are performed by flat antennas in a wireless manner, the other surface of the resonator can be remained uncoated. When the resonator is exposed to hydrogen gas, palladium film expands because of hydrogen absorption, causing the bending of the AT-cut crystal. The resonant frequency of an AT-cut resonator decreases as the bending curvature increases. ${ }^{28}$ Thus, the sensitivity in hydrogen-gas detection can be evaluated through the frequency decrease caused by the curvature change.

We first measured the sensitivity of our hydrogen-gas sensor with the as-deposited palladium thin film. Then, we performed the plasma treatment to the palladium-coated resonator for different exposure time, after which the hydrogengas detection measurements were performed again to evaluate the sensitivity enhancement. The morphology and structure change of palladium film before and after the plasma treatment were studied using AFM.

\section{Results and Discussion}

\subsection{Effect of plasma treatment on the sensitivity}

Figure 2 presents the responses of palladium-coated quartz resonator upon exposure to hydrogen gas of different concentrations. The palladium film thickness is $200 \mathrm{~nm}$. As can be seen in Fig. 2, the quartz resonator with as-deposited palladium film shows the lowest sensitivity. However, the sensitivity is significantly improved after the plasma treatment, and the total decrease in the resonance frequency increases with increasing the plasma exposure time. The steep frequency drop after the gas injection as shown in the enlargement of Fig. 2 will be attributed to the hydrogen attachment on the palladium surface, although a part of this drop was also caused

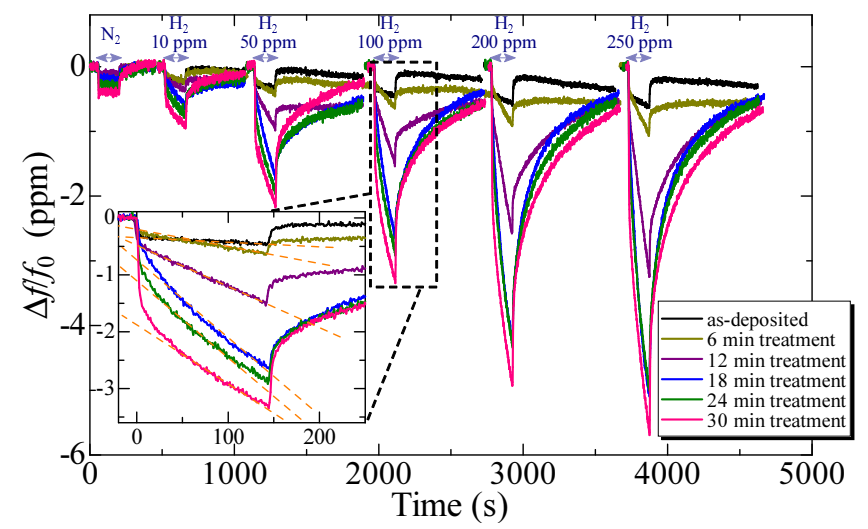

Fig. 2. Frequency responses of palladium-coated quartz-crystal resonator for injections of various concentration hydrogen gas with different plasma exposure times.

by the baseline shift at the gas injection because of additional injection pressure. This steep frequency drop is also remarkably enlarged after the plasma treatment, indicating that the surface status of palladium has been changed. Because a part of the initial frequency jump may be caused by the baseline shift due to the additional pressure, we focus on the frequency change after the jump which represents the hydrogen atoms migration process into palladium bulk. This part of frequency response can be approximated to a linear form, ${ }^{28}$

$$
\frac{\Delta f(t)}{f_{0}}=-A\left(k_{a} C_{H_{2}}+k_{d}\right) \cdot t
$$

where $f_{0}$ is the baseline resonance frequency, $A$ is a constant, $C_{H_{2}}$ denotes the hydrogen-gas concentration, $k_{a}$ and $k_{d}$ are reaction-velocity constants for absorption and discharge reactions.

We evaluate the hydrogen-gas concentration using the frequency slope after the steep drop, which is illustrated by the dashed line in the enlargement of Fig. 2. The relationship between the frequency slope and hydrogen-gas concentration is shown in Fig. 3(a), showing good linearity. It is clearly shown that the absolute value of the frequency slope increases with the increase of the plasma processing time. The effect of the plasma treatment appears to be saturated over $18 \mathrm{~min}$. The inset of Fig. 3(a) shows the relationship between the frequency slope and the hydrogen-gas concentration including the high concentration experiment after the plasma treatment for 30 min, demonstrating the good linearity between 10 and 1000 $\mathrm{ppm}$. Therefore, our sensor is capable of detecting hydrogengas in wide concentration range.

The reaction velocity constant for absorption $k_{a}$ is described by the Arrhenius law as $k_{a}=A_{0} \exp \left(-E / k_{B} T\right)$, with the activation energy $E$, Boltzmann constant $k_{B}$, and temperature $T$. By fitting a linear function to the measurements in Fig. 3(a), we extract the quantity $A k_{a}$ in Eq. (1) and calculate the absorption constant normalized that for the as-deposited palladium film. Fig. 3(b) shows the result. The absorption constant is significantly increased with the plasma-treatment time, and it saturates after 18 min exposure time. The increase in the velocity constant indicates reduction of the activation energy by the plasma treatment. 

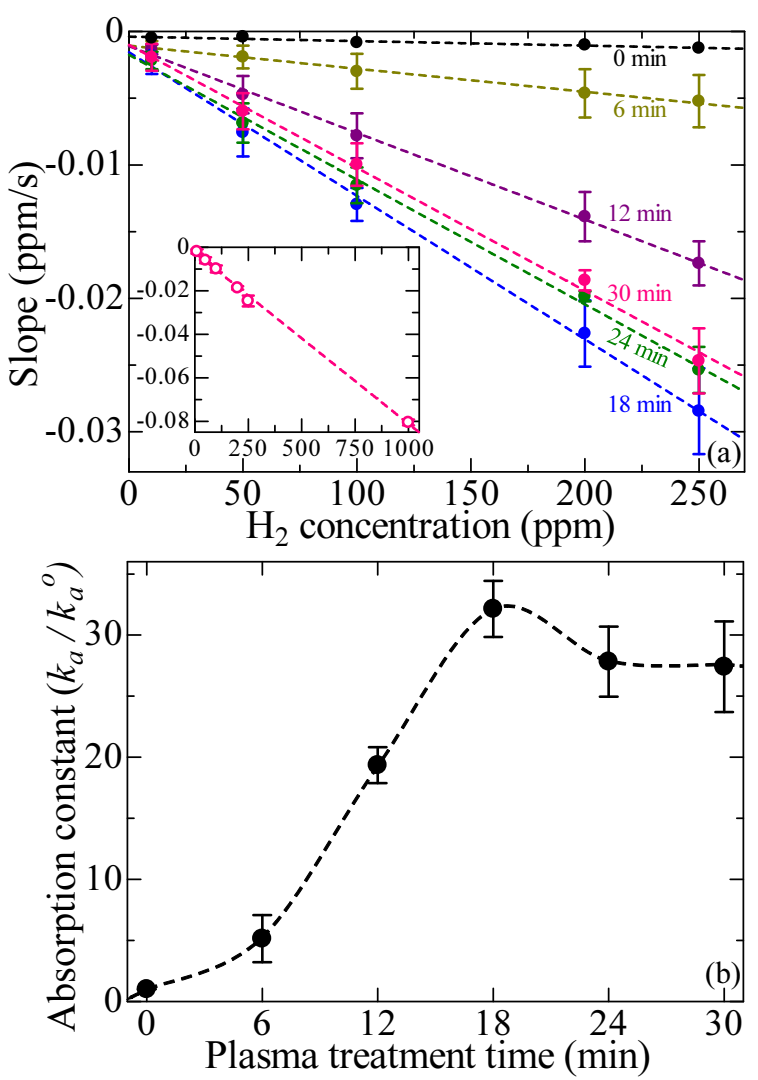

Fig. 3. (a) Relationships between the frequency slope and the hydrogengas concentration with various plasma exposure times. The number denotes the plasma exposure time in minutes. The inset shows the relationship including the high-concentration experiment $(1,000 \mathrm{ppm})$ with the sensor chip exposed to the plasma for $30 \mathrm{~min}$. (b) Dependence of the reaction velocity constant for hydrogen absorption on the plasma exposure time. $k_{a}^{0}$ denotes the velocity constant for the as-deposited palladium film.

\subsection{Palladium film morphology change by plasma treatment}

The AFM images of $200 \mathrm{~nm}$ palladium film shown in Fig. 4 have been taken in the as-deposited state and after the plasma treatment for $30 \mathrm{~min}$. The height image of the as-deposited palladium (Fig. 4(a)) consists of nearly equiaxial grains with less grain-size distribution. Its $R a$ roughness was about $3 \mathrm{~nm}$. The plasma treated palladium film, by contrast, exhibits finer grains with the $R a$ roughness of about $6 \mathrm{~nm}$. We observe some voids in the film. The increased roughness, decreased grain size, and generation of voids enlarge surface reaction area for chemical attachment, resulting in improvement of adsorption rate in the plasma treated palladium film. From the AFM phase images presented in Fig. 4(c) and (d), we can clearly observe the variation in grain size and grain boundaries. The average grain size of the as-deposited palladium film is about $100 \mathrm{~nm}$, and that of the plasma-treated palladium film is about $50 \mathrm{~nm}$. Compared with bulk diffusion, hydrogen atoms can show faster diffusion rate ${ }^{29}$ and higher solubility ${ }^{30}$ at grain boundaries, which should contribute to the sensitivity improvement: The grain structure gets finer through the plasma treatment, and the volume fraction of the grain boundary significantly increases, accelerating the hydrogen atom migration into palladium. The hydrogen absorption initially proceeds from active sites on palladium surface near lattice defects and successive hydrogen absorption-desorption leads to formation of more active sites. ${ }^{31}$ Not only grain boundaries, but also other lattice defects can be introduced by the plasma treatment. ${ }^{32}$ Point defects such as vacancies contribute to reduction in the activation energy of hydrogen diffusion and enhance the hydrogen solubility of the materials, ${ }^{12,33}$ which explains our experimental results in Fig. 3(b) as well.

The structure change discussed above will be the consequence of ion and electron bombardment. Existing studies have shown that the surface temperature of metal exposed to plasma can reach several hundred degrees Celsius. ${ }^{18,19}$ Thus, the post-annealing effect caused by the plasma treatment also contributes to recovery and recrystallization of the film. These palladium film structure changes significantly improve the performance of the hydrogen-gas sensor, allowing lower detection limit, faster response time, and faster recover time. The plasma treatment, thus, increases the sensitivity by a factor of 30 and allows detection of hydrogen gas with concentrations less than $1 \mathrm{ppm}$ in our sensor.

We also reveal that activation of the the hydrogen-gas sensor with a high-concentration hydrogen gas achieves the stable frequency response as shown in Fig. 2. Figure 5(a) presents the resonance-frequency change in a sequential injection of a 100-ppm hydrogen gas, where the resonant frequency fails to recover to the original baseline by flowing the high purity nitrogen gas after the hydrogen-gas injection. However, both frequency change and frequency recovery improve by repeating the hydrogen-gas measurement. Similar behavior was previously reported, ${ }^{10,11,34-36}$ but they did not clarify the detailed mechanism. In $\alpha$ phase palladium hydride, the linear expansivity $\epsilon$ is proportional to the hydrogen atom content $n$ inside palladium as ${ }^{37} \epsilon=0.026 \cdot n$. According to Sievert's law, ${ }^{38}$ the possible maximum hydrogen atom content is estimated to be $7.7 \times 10^{-4}$ at $100 \mathrm{ppm}$ hydrogen gas in the experiment of Fig. 5(a). The resulting strain in the Pd film should be $\sim 2 \times 10^{-5}$, which is far less than the strain for plastic deformation, and we do not expect plastic deformation during the repeated injection of Fig. 5(a). We also find that this unstable response can be eliminated by repeatedly injecting high concentration hydrogen gas (1000 ppm) and the highly purity nitrogen gas about four times as shown in Fig. 5(c). The experimental results in Fig. 2 was obtained after the"activation" by the high-concentration hydrogen gas. Therefore, the repeated exposure to the high concentration (1000 ppm) hydrogen gas doesn't affect the following detection of a low concentration (10 ppm) hydrogen gas injection.We suppose that by repeating such hydrogen charge and discharge procedure at a high concentration will form the "hydrogen path channels" from the surface to the bulk, and once such path has been created, the stable adsorption and discharge behavior is achieved.

\subsection{Thickness dependence of the sensitivity}

Because the average size of grains affected by the plasma treatment is $\sim 100 \mathrm{~nm}$, which is of the order of the thickness, we expect that the plasma treatment caused the structural change in the nearly whole region of the Pd thin film, improving the hydrogen storage ability. Besides, it will affect the surface chemical state of Pd: As shown in the inset of Fig. 2 , the frequency change takes two steps. The first steep frequency drop and the subsequent slower frequency decrease. 

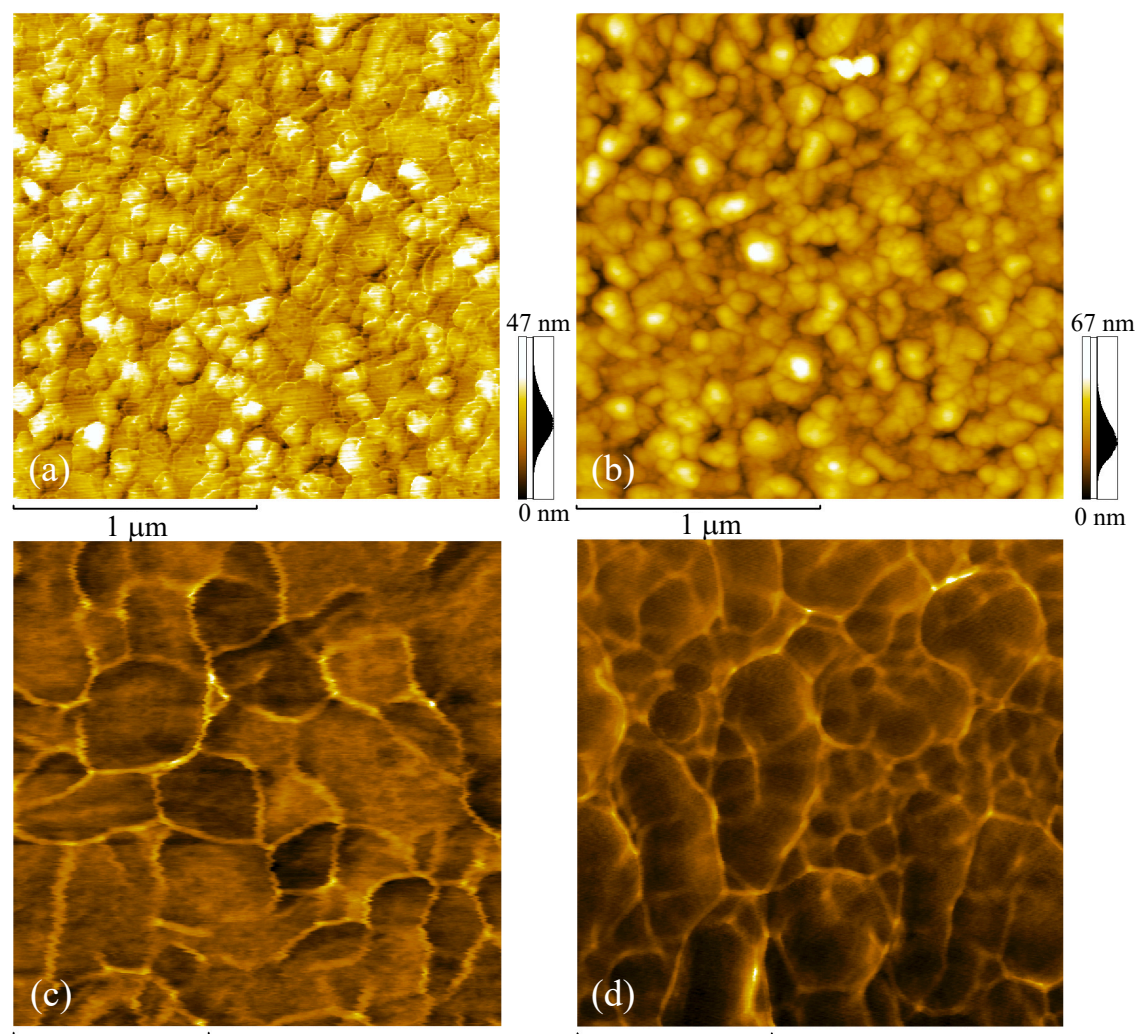

$200 \mathrm{~nm}$

$200 \mathrm{~nm}$

Fig. 4. AFM height images of as-deposited palladium film (a) and plasma treated palladium film (b); AFM phase images of as-deposited palladium film (c) and plasma treated palladium film (d).
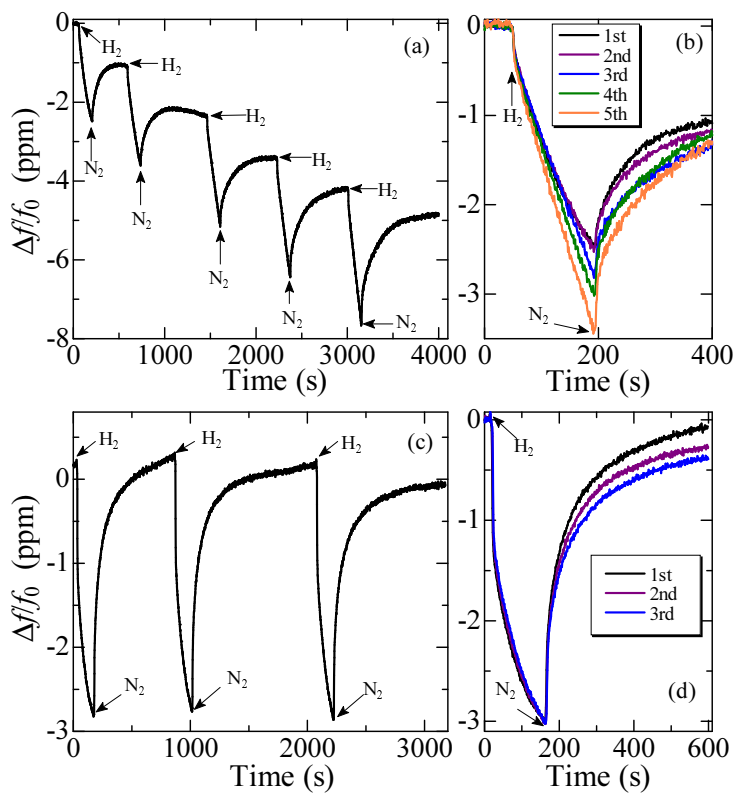

Fig. 5. (a) Frequency response of the palladium-coated resonator to the sequential injections of 100-ppm hydrogen gas, (b) comparison of the four measurements in (a), (c) the frequency response after the repeated highconcentration $(1,000 \mathrm{ppm})$ hydrogen-gas exposure process, and (d) comparison of the three measurements in (d).

Our previous study ${ }^{28}$ indicated that the first steep frequency drop was caused by the hydrogen attachment on the Pd surface. The plasma treatment enhances the first frequency drop
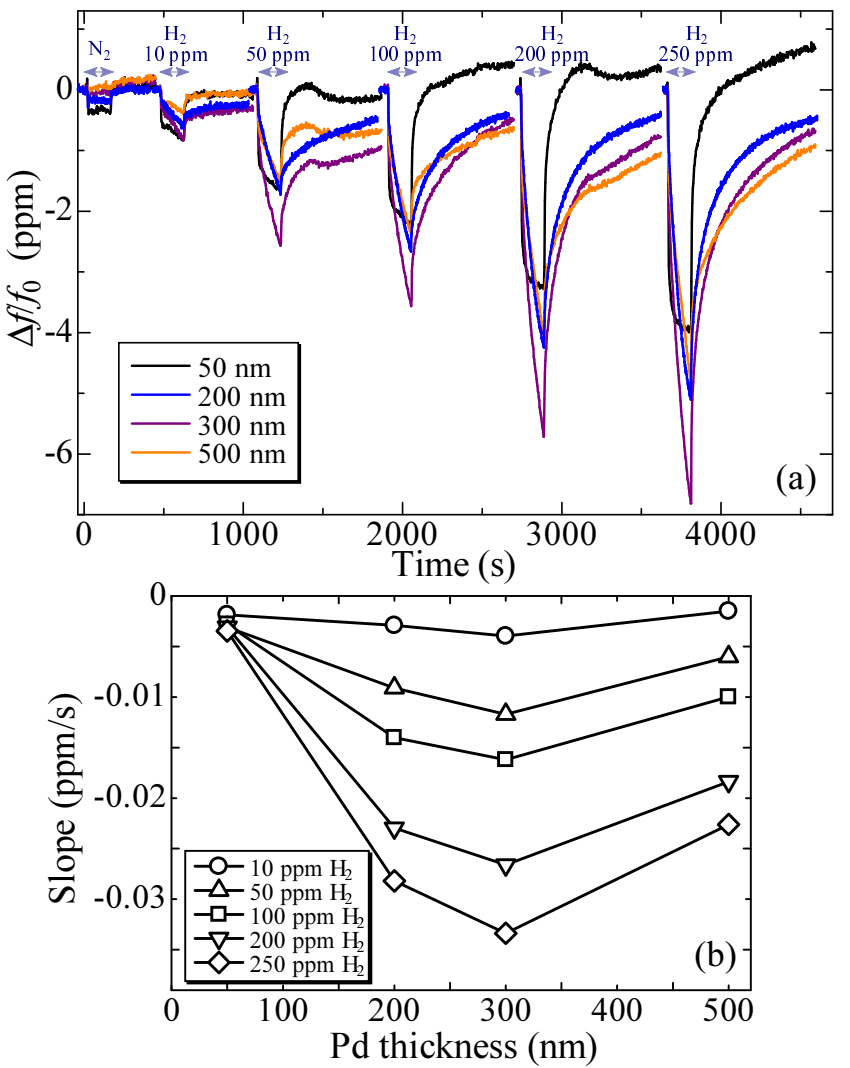

Fig. 6. (a) Frequency change and (b) sensitivity of quartz resonators coated with palladium films of various thicknesses upon hydrogen-gas exposure. 
as demonstrated in the inset of Fig. 2, indicating that it improves the hydrogen adsorption ability.

We deposited palladium thin films of various thicknesses (from $50 \mathrm{~nm}$ to $500 \mathrm{~nm}$ ) on single side of the quartz resonators to investigate the influence of the palladium thickness on the sensitivity in our hydrogen-gas sensor. The palladiumcoated resonators were treated in plasma for $18 \mathrm{~min}$ before the hydrogen-gas detection experiments. The frequency response with different palladium thicknesses are given in Fig. 6(a). When the thickness of palladium thin film increases from 50 $\mathrm{nm}$ to $300 \mathrm{~nm}$, the sensitivity (frequency slope) increases, which is in accordance with our expectation, because thicker film can cause larger curvature in the crystal upon the hydrogen absorption. Within the hydrogen exposure time in our experiments, the resonator with $500 \mathrm{~nm}$ palladium, however, shows smaller frequency change compared with $200 \mathrm{~nm}$ and $300 \mathrm{~nm}$ palladium films. We observed partial delamination of the $500 \mathrm{~nm}$ film, which released the residual stress caused by the hydrogen-gas absorption and deteriorated the sensitivity. Fig. 6(b) shows the relationship between the sensitivity (frequency slope) and the palladium thickness, revealing the optimum thickness of $300 \mathrm{~nm}$.

\section{Conclusion}

We present a method to improve the sensitivity of palladium based hydrogen-gas sensor by plasma treatment. The sensitivity enhancement was confirmed using a wireless quartz-crystal resonator. Palladium thin film was deposited on single surface of quartz crystal resonator. The frequency decrease of resonator caused by geometry change was utilized to evaluate the sensitivity of hydrogen gas detection. We reveal that the sensitivity increases, takes a maximum, and becomes stable as the plasma-treatment time increases, indicating the optimum exposure time. Through the analysis of palladium film using AFM, we attribute the enhancement of the sensitivity to the morphology change and post-annealing effect in palladium film induced by the plasma treatment. The higher roughness and finer grain size of plasma-treated palladium film enlarge the effective surface area for hydrogen gas attachment. The increase in volume fraction of grain boundaries and other defects improves the hydrogen atom diffusion speed. Compared with as-deposited palladium film, the hydrogengas detection sensitivity is improved by a factor of about 30 after plasma treatment.

\section{Acknowledgement}

This research was supported by Development of Advanced Measurement and Analysis Systems from Japan Science and Technology Agency (Project No. JP-MJSN16B5).

1) K. Yamanaka, S. Akao, N.Takeda, T.Tsuji, T. Oizumi, and Y. Tsukahara, Jpn. J. Appl. Phys. 56, 07JC04 (2017).

2) K. Yamanaka, S. Akao, N.Takeda, T.Tsuji, T. Oizumi, H. Fukushi, T. Okano, and Y. Tsukahara, Jpn. J. Appl. Phys. 58, SGGB04 (2019).

3) K. Yu, X. Tian, X. Wang, F. Yang, T. Qi, and J. Zuo, Sens. Actuat. B, Chem. 299, 126989 (2019).
4) T. Abe, N. Iwata, T. Tsuji, T. Mihara, S. Akao, K. Noguchi, N. Nakaso, D. Sim, Y. Ebi, T. Fukiura, H. Tanaka, and K. Yamanaka, Jpn. J. Appl. Phys. 46, 4726 (2007).

5) S. Okuyama, Y. Mitobe, K. Okuyama, and K. Matsushita, Jpn. J. Appl. Phys. 39, 3584 (2000).

6) B. Sharma and J. Kim, Int. J. Hydrog. Energy 42, 25446 (2017).

7) N. Nakamura, T. Ueno, and H. Ogi, Appl. Phys. Lett. 114, 201901 (2019).

8) K. Yoshimura, Y. Yamada, M. Okada, M. Tazawa, and P. Jin, Jpn. J. Appl. Phys. 43, L507 (2004).

9) K. Yamanaka, S. Ishikawa, N. Nakaso, N. Takeda, D. Y. Sim, T. Mihara, A. Mizukami, I. Satoh, S. Akao, and Y. Tsukahara, IEEE Trans. Ultrason. Ferroelectr. Freq. Control 53, 793 (2006).

10) J. F. Patton, S. R. Hunter, M. J. Sepaniak, P. G. Daskos, and D. B. Smith, Sens. Actuat. A, Phys. 163, 464 (2010).

11) R. Gremaud, M. Slaman, H. Schreuders, B. Dam, and R. Griessen, Appl. Phys. Lett. 91, 231916 (2007).

12) A.V. Panin, M.S. Kazachenok, O.M. Kretova, O.B. Perevalova, Y.F. Ivanov, A.M. Lider, O.M. Stepanova, M.H. Kroening, Appl. Surf. Sci. 284, 750 (2013).

13) F.D. McDaniel, F.U. Naab, O.W. Holland, M. Dhoubhadel, L.J. Mitchell, and J.L. Duggan, Surf. Coat. Technol. 201, 8564 (2007).

14) H. Abe, H. Uchida, Y. Azuma, A. Uedono, Z.Q. Chen, and H. Itoh, Nucl. Instrum. Meth. Phys. Res. B 206, 224 (2003).

15) Deepti, H. Kumar, A. Tripathi, A.B. Dey, M. Gupta, R. Krishna, and D.K. Avasthi, Sens. Actuat. B, Chem. 301, 127006 (2019).

16) J-H. Kim, A. Mirzaei, H.W. Kim, P. Wu, and S.S. Kim, Sens. Actuat. B, Chem. 293, 210 (2019)

17) P.K. Chu, J.Y. Chen, L.P. Wang, and N. Huang, Mater. Sci. Eng. R-Rep. 36, 143 (2002)

18) D. Wandke, M. Schulze, S. Klingner, A. Helmke, and W. Viöl, Surf. Coat. Technol. 200, 700 (2005).

19) D.N. Tran, V.P. Nguyen, T. Sakaki, T. Kikuchi, and N. Harada, Jpn. J. Appl. Phys. 50, 036202 (2011).

20) H. Ogi, Proc. Jpn. Acad., Ser. B 89, 401 (2013).

21) H. Ogi, K. Motohisa, T. Matsumoto, K. Hatanaka, and M. Hirao, Anal. Chem. 78, 6903 (2006).

22) H. Ogi, H. Nagai, Y. Fukunishi, M. Hirao, and M. Nishiyama, Anal. Chem. 81, 8068 (2009).

23) K. Noi, M. Iijima, S. Kuroda, and H. Ogi, Sens. Actuat. B, Chem. 293, 59 (2019).

24) K. Noi, A. Iwata, F. Kato, and H. Ogi, Anal. Chem. 91, 9398 (2019).

25) F. Kato, H. Noguchi, Y. Kodaka, N. Oshida, and H. Ogi, Jpn. J. Appl. Phys. 57, 07LD14 (2018).

26) H. Ogi, M. Fujii, N. Nakamura, T. Shagawa, and M. Hirao, Appl. Phys. Lett. 90, 191906 (2007).

27) L. Zhou, N. Nakamura, A. Nagakubo, and H. Ogi, Proc. 40th Symp. Ultrason. Electron. 40, 2P2-13 (2019).

28) L. Zhou, N. Nakamura, A. Nagakubo, and H. Ogi, Appl. Phys. Lett. 115, 171901 (2019).

29) H. Natter, B. Wettmann, B. Heisel, and R. Hempelmann, J. Alloys Compd. 253-254, 84 (1997).

30) U. Stuhr, H. Wipf, T.J. Udovic, J. Weissmuller, and H. Gleiter, J. Phys.: Condens. Matter 7, 219 (1995).

31) R. Nowakowski and R. Duś, Langmuir 19, 6750 (2003).

32) M. Y. Um, I. S. Jeon, D. I. Eom, and H. J. Kim, Jpn. J. Appl. Phys. 43, 4114 (2004).

33) V. I. Lavrent'ev, A. D. Pogrebnyak, A. D. Mikhalev, N. A. Pogrebnyak, R. Shandrik, Z. Zecca, and Y. V. Tsvintarnaya, Tech. Phys. Lett. 24, 334 (1998).

34) D.R. Baselt, B. Fruhberger, E. Klaassen, S. Cemalovic, C.L. Britton, S.V. Patel, T.E. Mlsna, D. McCorkle, and B. Warmack, Sens. Actuat. B, Chem. 88, 120 (2003).

35) Z. Hu, T. Thundat, and R. J. Warmack, J. Appl. Phys. 90, 427 (2001).

36) M-W. Lee and R. Glosser, J. Appl. Phys. 57, 5236 (1985).

37) W. M. Mueller, J. P. Blackledge, and G. G. Libowitz, Metal Hydrides (Academic, New York, 1968), Chap. 12.

38) A. Sieverts and W. Krumbhaar, Ber. Dtsch Chem. Ges. 43, 893 (1910). 